\title{
OaSis: An Application Specific Operating System for an Embedded Environment
}

\begin{abstract}
Portable and affordable assistive devices are calling necessities for the physically challenged population putting forth the requirement of designing embedded systems tuned to specific assistive applications. In this paper, we present a low footprint embedded operating system, OaSis, which forms the core of embedded devices being developed for the speech impaired, the visually impaired and those with neuro-motor disorders. The target hardware, housing OaSis and on which the applications are ported is Geode GX1-233 processor based POS-563 of Advantech. The system is presently being ported to more energy efficient ARM based platform. This system currently supports three applications: Shruti - a Vernacular speech interface, Sanyog - a Visual Communication interface and a web browser. The OaSis embedded operating system has been optimized to $90 \mathrm{~KB}$ and provides all the features required for the specific applications. The different modules of OaSis have been described and have been compared with other operating systems.
\end{abstract}

\section{Introduction}

Assistive Technology [1, 5] deals with development of hardware and software that enables physically challenged people to partially overcome the hindrances to mobility, communication and other allied difficulties. Many of the modern assistive devices are computer based $[2,3]$. However, for better usage, it is required that the systems be on low cost portable platforms instead of being executed on PCs or laptops. The affordability issue is all the more important for the people of the developing countries, since most of the portable systems are costly with respect to these target users. The portability issue therefore calls for indigenous development of low cost embedded assistive systems. 
The design or selection of suitable operating system kernel forms a basic need of such embedded system design. This paper presents OaSis, a low footprint kernel designed for such embedded applications. The hardware platform, on which the kernel and the applications were first developed and ported, is a Geode processor based Advantech board. While this exercise served the purpose of proof of concept, we are now porting it to ARM processor based platforms to result in power efficient portable system. The applications that have been developed around OaSis, include a text-to-speech system to be used by the visually handicapped -Shruti, a visual communication system to be used as an Augmentative and Alternative Communication System (AAC) $[4,5,6]$ for use of the people affected with cerebral palsy, speech impairment and motor neuron disorders Sanyog. In addition, a Web Browser application is included for accessibility to the internet.

This paper concentrates on the design of OaSis and also presents some of the embedded applications developed around OaSis.

The paper is organized as follows.

Section 2 presents the overview of the target hardware platform and the kernel of the OaSis operating system. The next section discusses the application programming interface (API) provided with OaSis. Section 4 describes the device drivers. Section 5 discusses the manner in which $\mathrm{OaSis}$ has been optimized for targeted applications. Section 6 presents a comparison of OaSis with other operating systems. Section 7 describes the applications, which have been ported on OaSis. The next section concludes the paper.

\section{Overview of OaSis and the Hardware Platform:}

The aim of OaSis is to provide an optimized low footprint operating system for an embedded environment running a few specific applications, as mentioned above.

The major components of OaSis nanokernel are shown in Fig. 1.

The current system consists of the OaSis nanokernel, a modified form of the UIP TCP/IP stack [8], emulation API's providing the low-level system functionality required by the applications and drivers for the following: clock, ATAPI, Ethernet, LCD, keyboard and sound blaster. 


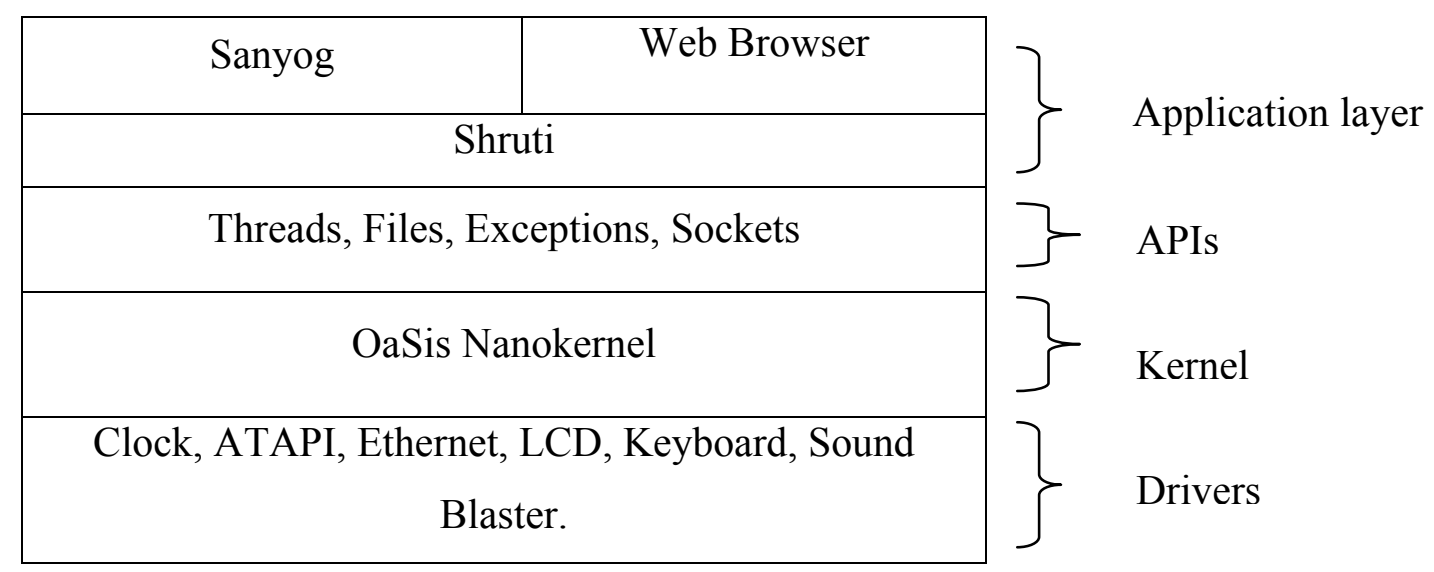

Figure 1: OaSis nanokernel with components

\subsection{The Hardware Platform - POS - 563 Port}

\subsubsection{The POS - 563}

The POS-563 is a low cost, fan-less Geode GX1-233 board specially designed for POS applications. The GX1-233 processor allows for fan-less operation that virtually eliminates heat buildup problems .The POS-563 has one PCI/ISA expansion slot, four digital I/Os and four on-board serial ports each with $+5 \mathrm{~V} /+12 \mathrm{~V}$ power. The POS-563 uses a standardized layout based on Western Digital's LPM/LPX form factor. It is $100 \%$ PC compatible and ready for any existing PC software or hardware. Other on-board industrial features not found on conventional mother-boards include a watchdog timer for dependability during unmanned operations, and CMOS backup to Flash ROM. The onboard SSD socket can also be used to support DiskOnChip and Flash modules.

\subsubsection{The POS - 563 Port}

In preparation for working with real POS-563 hardware, a port of the Geode GX1-233 was made using 32-bit GNU C compiler and GNU inline assembler.

Figure 2 shows a broad macro view of the OaSis. The arrows and the direction show flow of data/information. The different units of the OS are shown distinctly for convenience of illustration, though in actual operation they may be overlapping. The kernel, though shown separately to indicate that it is the one that primarily executes the threads, infact encompasses the threads. Further the system boots from the compact flash 
using the grub boot loader. Each application is run on the system as a thread. The system uses the standard Ext2 file system to read and write to the compact flash. As the system is very much application specific, it has only those drivers that are required to run the above mentioned applications. As such it has drivers only for the Sound Blaster (for the Shruti), the Realtek Ethernet (for the Web Browser), the serial UART (for the Sanyog), the LCD and the keyboard.

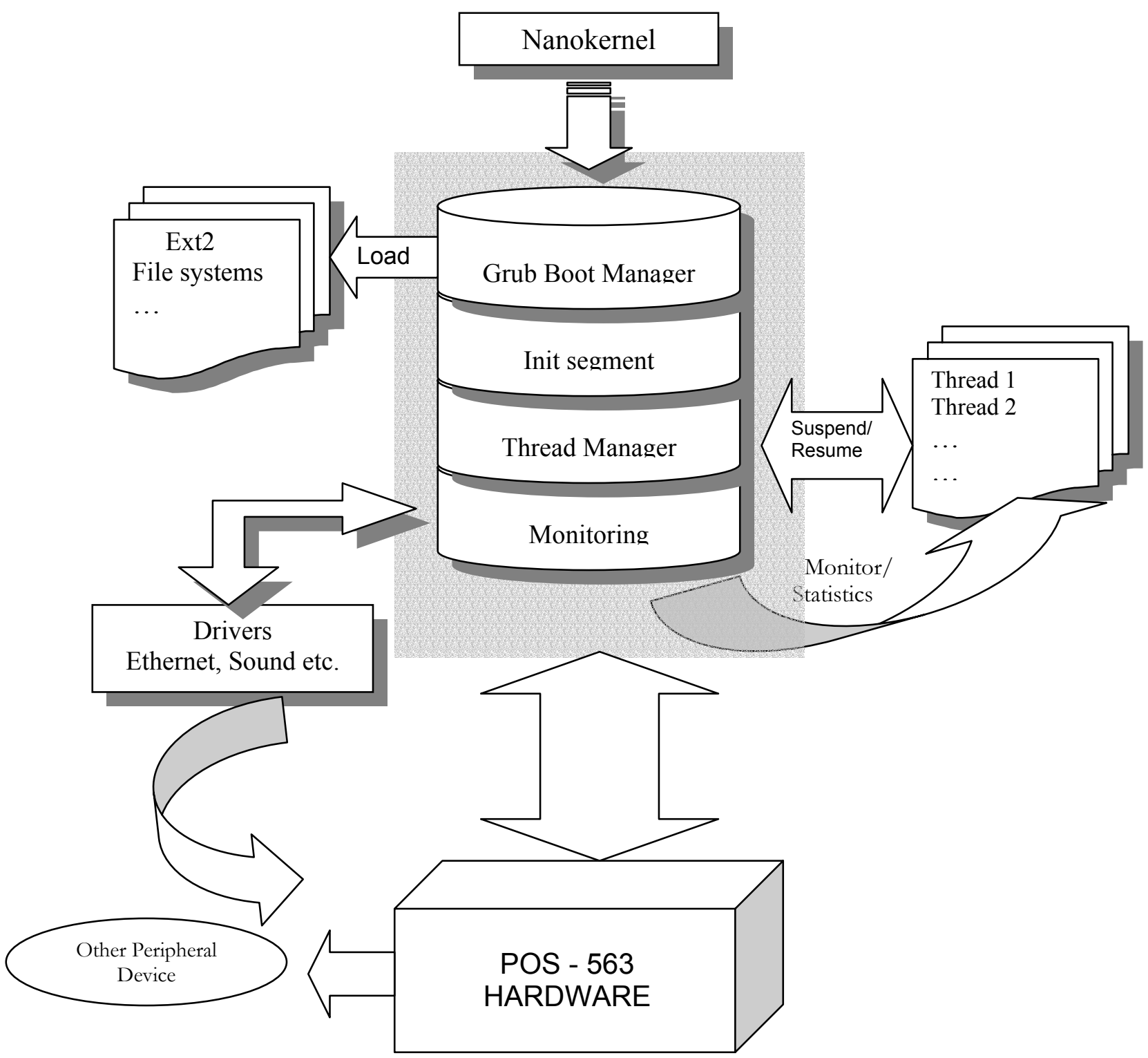

Figure 2: Illustration of the OaSis OS 


\subsection{OaSis Nanokernel}

The OaSis kernel has most of the characteristics of a standard embedded OS kernel i.e. interrupt support and soft Real-Time scheduling. The scheduler and the interrupt control are closely interwoven. The data structures are highly optimized to allow the full kernel to reside in memory. OaSis, a modified subset of Montague's JN nanokernel [7] follows a classic soft-real-time architecture informally known as a Cutler kernel. The present kernel consists of a single work-loop, driven by a queue of control blocks. The kernel begins execution in response to an interrupt. Once started, the kernel continues to execute until no control blocks remain in the queue. Each control block contains the address of a routine which the kernel must execute. Historically, these routines have been called fork routines in the Cutler kernels. Since fork routines cannot block and must have a bounded execution time, they have many characteristics of routines written for a hardreal-time environment. Neither the fork routines nor the kernel need to use explicit mutual exclusion to access global data structures, reducing the need for explicit synchronization. The OaSis currently supports Ext2 file system [9]. For networking the OaSis supports Ethernet [11]. Although we adopted well-known kernel architecture a full kernel was not implemented. The implemented subset is referred to as a nanokernel. Although the term has been disparaged, it is believed to be warranted [13].

OaSis is a very low footprint embedded operating system; in fact the current OS image size is well within 90KB including all the modules i.e. the nanokernel itself, the various application interfaces and the different device drivers, excluding the database required for running the speech application, Shruti.

\section{The Application Programming Interface (API)}

This section documents the API required by the application to execute on OaSis. The API is divided into 4 classes: thread, graphics, file and inter process communication.

\subsection{Thread APIs}

The threading API routines are conventional and were suitably modified from the JN nanokernel [10]. Thread priorities range from 0 to 16. This high range of thread priorities is required for a soft Real Time environment to provide the developer with a larger range, 
so as to effectively decide the priorities of his applications. A sysThreadCreate() API routine creates a thread in suspended state, sysThreadExit() can terminate any thread, and sysThreadYield() rotates the threads at the current priority level. Any thread can be removed from scheduling consideration by sysThreadSuspend() and restored to scheduling eligibility by sysThreadResume(). The unique integer thread ID of the caller can be obtained as well as the priority of any thread can be set and read.

\subsection{File APIs}

Though the file system used by the OaSis embedded operating system is a subset of the Ext2 file system, all the standard conventions have been followed and the standard data structure for the inode and the file descriptor table has been implemented. The superblock structure also remains the same. However, there have been some changes made keeping in mind the application specific nature of our operating system.

The text to speech application, Shruti produces the output speech file by concatenating small wav files, which are read from a database. So to make the text to speech application run in an optimized way, OaSis needs to search through the database very quickly and also read from and write to a file quickly. The same requirement exists for the application Sanyog. The usual way to search through a directory for a file is linear search strategy. But OaSis uses a binary search strategy when searching for files within a directory to quicken the search. As far as the API functions are concerned there is the standard open, close, read, write and lseek functions, which have all been implemented keeping in mind the application's requirement. By which it is meant that we have departed from the standard implementation wherever necessary to skip a few non required steps.

\subsection{Graphics APIs}

The Sanyog Application needs a grid type layout for displaying its icons. A user will select a set of icons from the display with switches attached to the serial port. More details about the Sanyog application can be found in section 7.1. OaSis being an application specific OS, displaying graphics in a fast and optimized way was one of the major concerns. The graphics API has a simple Java AWT type layout manager. The API 
consists of the TextClass, GraphicsClass, LayoutBoxClass and GridLayoutClass. The GridLayoutClass extends the LayoutBoxClass and divides the display area into grids, where each grid box can contain a graphics object or text object or both. The LayoutBoxClass takes into account the graphics and text object attached to the box, and displays the object in the grid box whenever the display_object function is called. The TextClass and GraphicsClass read and buffer the text and graphics object respectively. The system currently can display portable pixel map (PPM) format

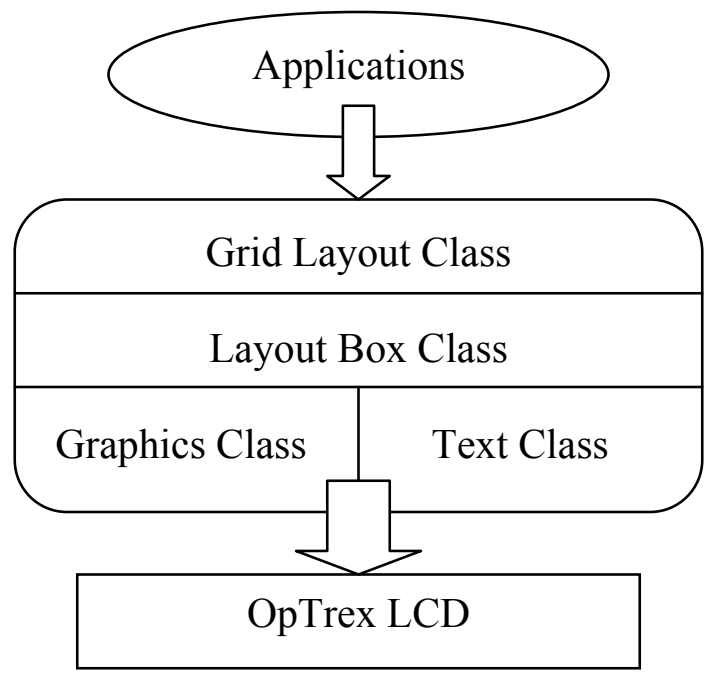

Figure 3: The Graphics API files.

\subsection{Inter Process Communication (IPC) APIs}

The Web Browser application downloads a web page from the internet and then applies a transcoding algorithm to convert the page, amenable to presentation on the limited display capability of the client device. The transcoded content is then passed to the text to speech application, Shruti. Inter process communication is required in OaSis when one realizes that the Shruti application and the browser need to communicate between themselves. Similar communication is required between Sanyog and Shruti. Considering the above mentioned need, a simple but fast implementation of the Pipe message passing data structure was created.

\section{Drivers}

We have currently implemented device driver for the serial UART, the Keyboard and the Sound Blaster. In addition we have implemented a driver for a Realtek 8139c Ethernet card. Following is a short description of the drivers:

- Realtek Ethernet: This driver has been implemented in OaSis for the Web Browser application. This driver has two major components: 
a) Packet Transmission: The transmit path of RTL8139 uses four descriptors, each descriptor having a fixed IO address offset. The four descriptors are used in round-robin fashion. As a descriptor is written, the PCI operation starts and moves a packet from the memory, specified by the descriptor, to the Transmit FIFO. The Transmit FIFO is a $2 \mathrm{~K}$ bytes buffer in the chip that holds the data prepared to be send to the line (cable). Data in Transmit FIFO starts moving to the line when early transmit threshold is meet. Early transmit threshold is also specified in the descriptor.

b) Packet Reception: The receive path of RTL8139 is designed as a ring buffer. Data coming from line is first stored in a Receive FIFO in the chip, and then moved to the receive buffer when the early receive threshold is met. The status of receiving a packet is stored in front of the packet (packet header).

- $\quad$ Sound blaster: The need for this driver arose due to the text to speech application Shruti. The driver uses DMA to transfer the sound file to a buffer where from the DSP chip processes it to get the sound.

- Keyboard: This driver is used by all applications. Implementation uses the standard approach i.e. with a circular queue and a process queue for the device.

- Serial UART: The need for this driver came because in the iconic application, Sanyog a user can give input to the application through special access system. In OaSis we have special external switches connected through the serial port. These switches are needed for the people with neuro-motor disabilities, who cannot use the standard input devices.

\section{Optimization of OaSis:}

OaSis has been optimized in many ways for the three applications for which it has been implemented. The thread, work blocks and memory data structures have been highly optimized keeping in mind that only a few applications will be running at the same time. The thread data structures are very light, thus allowing the scheduling to proceed rapidly. Only those drivers have been implemented which are necessary for these specific applications. The file system and the file APIs have been modified in several places. As the speech application requires to read from a large database, a binary search strategy has been implemented so that search through a directory for a file is faster. Sanyog uses a 
grid type interface to display the icons and also requires displaying the same icon more than once at times. The Graphics API has been written considering the display constraints of an embedded device. The Graphics API has very simple data structures in which it reads a graphics object and buffers it. This helps in a much faster display of icons whenever necessary.

\section{Comparison}

In this section we present a comparison of OaSis with respect to other standard operating systems.

\subsection{System size}

Figure 4 gives a comparison of the storage requirements for various embedded operating systems with the OaSis operating system which clearly shows the difference in size achieved by OaSis.

\begin{tabular}{|c|c|c|c|c|}
\hline OaSis & Embedded XP & Embedded Linux & CE.NET & Micro Linux \\
\hline $90 \mathrm{~KB}$ & $100 \mathrm{MB}$ & $16 \mathrm{MB}$ & $16 \mathrm{MB}$ & $512 \mathrm{~KB}$ \\
\hline
\end{tabular}

\section{Figure 4: Storage Requirements}

\subsection{Context Switch Time}

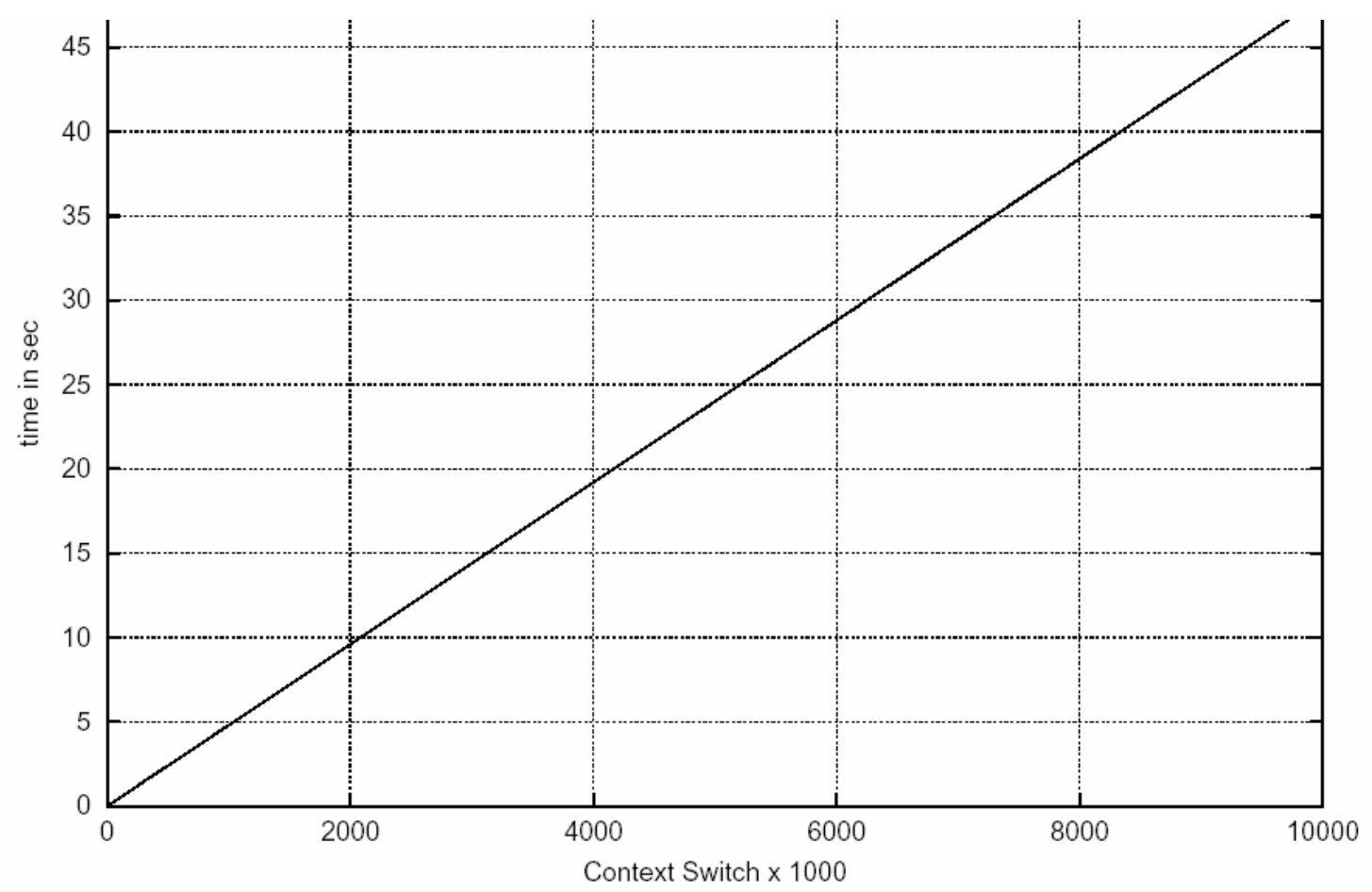

Figure 5: time in sec (y axis) vs. context switches x 10000 (x axis). 
The values for context switch time for OaSis were obtained for a set of discrete points and the near linear relationship obtained is plotted in Figure 5. Operating systems like RH - 7.2, Windows XP, Windows 2000 SP2 have a context switching time which varies with the load and ranges from a minimum of $3 \mu \mathrm{sec}$ for Win $2 \mathrm{~K} \mathrm{SP} 2$ and maximum of $14 \mu \mathrm{sec}$ for Red Hat 7.2 Linux. This data was collected on a TP600X, $650 \mathrm{MHz}$ by Bradford [12]. In comparison, our system has a context switching time of approximately $5 \mu \mathrm{sec}$, which seems pretty good for our embedded applications.

\section{Applications:}

\subsection{Sanyog - Visual Communication Interface}

The iconic communication application provides facilities to physically challenged people to communicate normally with others. The application has a very simple GUI through which user selects icons. It has the facility to form natural language sentences with various moods, tenses and also interrogative sentences.

The salient features of the system are:

- Displaying relevant icon set in front of the user to reduce icon selection time.

- A feedback mechanism in the selection process to give the user chance to rectify selection error.

- Generating various types of natural language sentences from the selected icons. The system at its present state forms only simple sentences with following variations: Assertive sentences, Simple negative sentences, Sentences with different tenses, Sentences with different

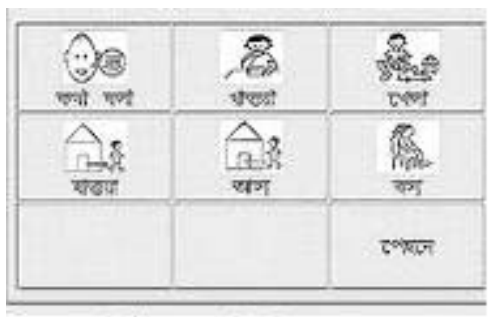

Figure 6: Sanyog moods and Interrogative sentences.

- $\quad$ Facility to store and display frequently used or user generated sentences.

- The application is equipped with speech synthesis technique to utter generated or stored sentences.

- The application can be integrated with special input devices other than standard input devices (mouse, keyboard). This facilitates disabled user to operate the system with less effort.

- A data entry interface to enter data and image required by the system. 


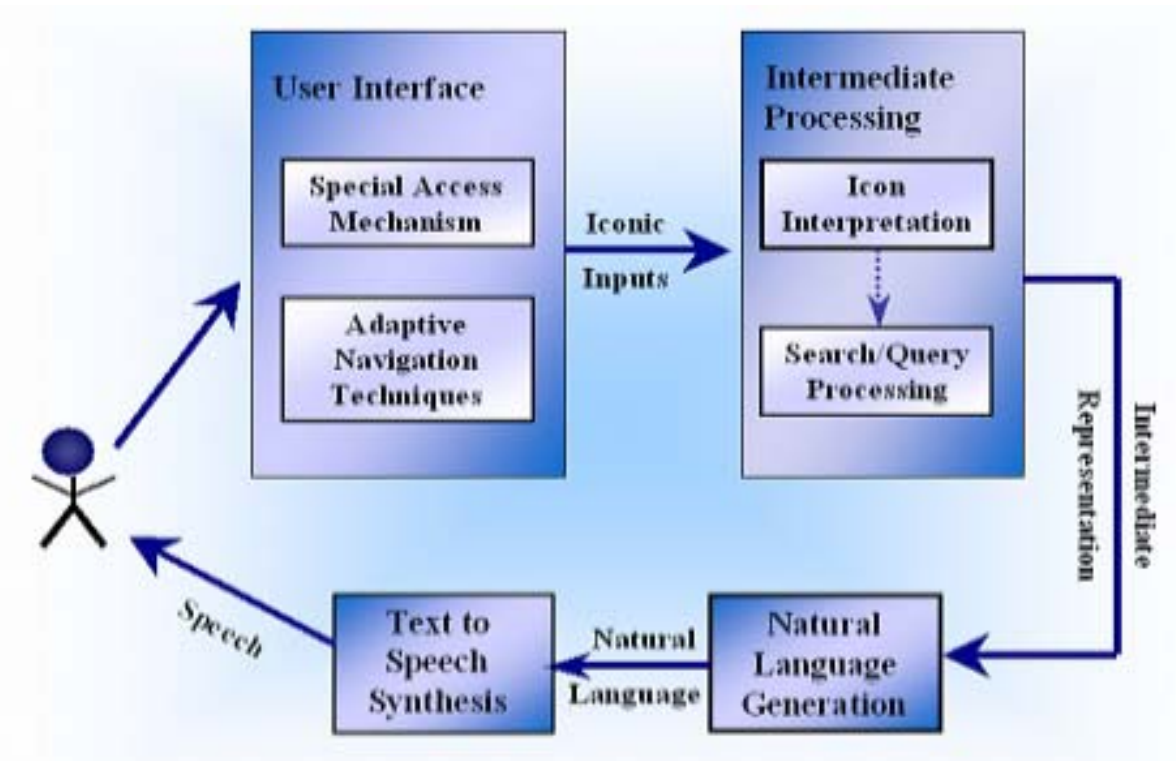

Figure 7: General Architecture of Sanyog

In figure 7 the general architecture of Sanyog is shown. A user can give input to the application through special access system, other than normal input devices like keyboard and mouse. Adaptive navigation has been used to reduce the time and effort spent by the user during access or selection of icons. The icons selected are interpreted by the Icon Interpretation module and then passed to the Natural Language Generation module. The generated sentence is then fed to the text to speech application.

\subsection{Shruti - Vernacular Speech Interface}

The Text to Speech (TTS) application acts as a computer interface for the visually challenged, for whom graphical interfaces are not viable. It also serves the purpose of the voice of the speech impaired. The TTS is based on a concatenation of speech approach. The building blocks of concatenative synthesizers are the signal units that are concatenated, in this cases partnemes and phonemes. The units have been spliced out from prerecorded utterances taking into consideration the combination of all vowels and consonants. Given a sentence in text, the synthesized speech will be generated after some steps. In the first stage it will be analyzed by a Natural Language Parser. After Morphological and Phonological analysis, the grapheme string is converted to a phoneme string which can be directly mapped to the partneme dictionary and concatenated. 
The method described above will generate synthetic speech but the real challenge is to make it sound natural. Rather than keeping a constant frequency for all utterances it is allowed to vary randomly within a small limit (typically 3-4\%) to make it sound more natural. This is called jitter. The way of utterance, i.e. the intonation and prosodic variations are an integral part of natural speech. For modeling intonation and prosody on the DSP end, the pitch, intensity and duration of phonemes are controlled by parameters. A detailed analysis of all the algorithms used to make a natural sounding speech synthesis system is beyond the scope of this section.

\subsection{Web Browser}

The Web Browser is a very low footprint simple embedded browser. It uses a set of routines which take input (key strokes or mouse clicks) from a user, use TCP/IP to perform Web transactions, and send the results to an output device, the LCD display. The application can also make direct calls (calls not initiated by an input device) to the browser to perform such actions as loading a specific HTML file.

\section{Conclusion:}

A working network computer with this custom operating system has been implemented for a 32bit PC, the POS-563. The requirements of this system were predefined by the range of applications. The system has been ported to a Geode GX1-233 processor based Advantech POS-563 board. While this exercise served the purpose of proof of concept, we are now porting it to ARM processor based platforms to result in power efficient portable system. The paper describes a novel operating system so that the specified applications would perform better in this custom OS than in a standard OS. The system is reasonably robust and can serve as a test bed for future work. It was shown that it is possible to do TCP/IP network development and related research at a very low cost. The applications have been ported and their successful execution proves the efficacy of OaSis.

\section{References}

[1]. Proceedings of the ACM SIGCAPH Conference on Assistive Technologies, 2000. 
[2]. Newell Morris A, Booth L, and Ricketts I, Syntax PAL: A writing aid for languageimpaired users, in ISAAC-92. [Also in Augmentative and Alternative Communication, Vol.8, 1992].

[3]. Pennington C.A.and McCoy K.F, Providing Intelligent Language Feedback for Augmentative Communication Users, in Assistive Technology and Artificial Intelligence, Applications in Robotics, User Interfaces, and Natural Language Processing, (Mittal V.O et al eds.), LNAI 1458, Springer Verlag, 1998

[4]. K. Rumble Gilian and Larcher Janet, AAC Device Review, Vocation Pub. 1998

[5]. Vanderheyden P B.and Pennington C.A, An augmentative communication interface based on conversational schemata, in Assistive Technology and Artificial Intelligence, Applications in Robotics, User Interfaces, and Natural Language Processing, (Mittal V.O et al eds.), LNAI 1458, Springer Verlag, 1998.

[6]. Warrick A and Kaul Sudha, Their Manner of Speaking, Indian Institute of Cerebral Palsy, Kolkata, India, 1997

[7]. B. R. Montague. JN: OS for an embedded Java network computer. IEEE Micro, 17(3):54-60, May/June, 1997.

[8]. Adam Dunkels. uIP - A Free Small TCP/IP Stack. Technical report, http://dunkels.com/adam/uip/.

[9]. Rémy Card, Theodore Ts'o and Stephen Tweedie. Design and Implementation of the Second Extended Filesystem. The First Dutch International Symposium on Linux, ISBN 90-367-0385-9.

[10]. B. R. Montague. JN external API. Technical report, U. of Calif. Santa Cruz, UCSCCRL-97-17, 1997.

[11]. Realtek Semiconductor Corporation. Realtek 3.3V single chip fast Ethernet Controller with power management RTL8139C (L). Revision 1.1, November 1999.

[12]. Dr Edward Bradford. High performance programming techniques on linux and windows. http://www-106.ibm.com/developerworks/linux/library/l-rt9/. July 1, 2002.

[13]. Jochen Liedtke. Towards real microkernels. Communications of the ACM, 39(9):70-77, September 1996. 